\title{
G-BA FÖRDERT PROJEKT „MUMI“
}

Mundgesundheit von Migranten-- Das Hamburger Projekt „MuMi“ („Förderung der Mundgesundheitskompetenz und Mundgesundheit von Menschen mit Migrationshintergrund") bezieht eine Förderung des Innovationsausschusses des Gemeinsamen Bundesausschusses (G-BA). Das Projekt wird gemeinsam von der Poliklinik für zahnärztliche Prothetik und dem Institut für Medizinische Soziologie am Universitätsklinikum Hamburg-Eppendorf (UKE) verantwortet. Ziel ist es, die Mundgesundheit von Migranten unter Berücksichtigung kulturspezifischer Indikatoren sowie Risikofaktoren zu durchleuchten. Außerdem wird ein mehrsprachiges, sekundärpräventives Schulungsprogramm zur Steigerung der Mundgesundheitskompetenz und Mundgesundheit erprobt. „MuMi“ wird mit 744.000 Euro vom G-BA gefördert. JG

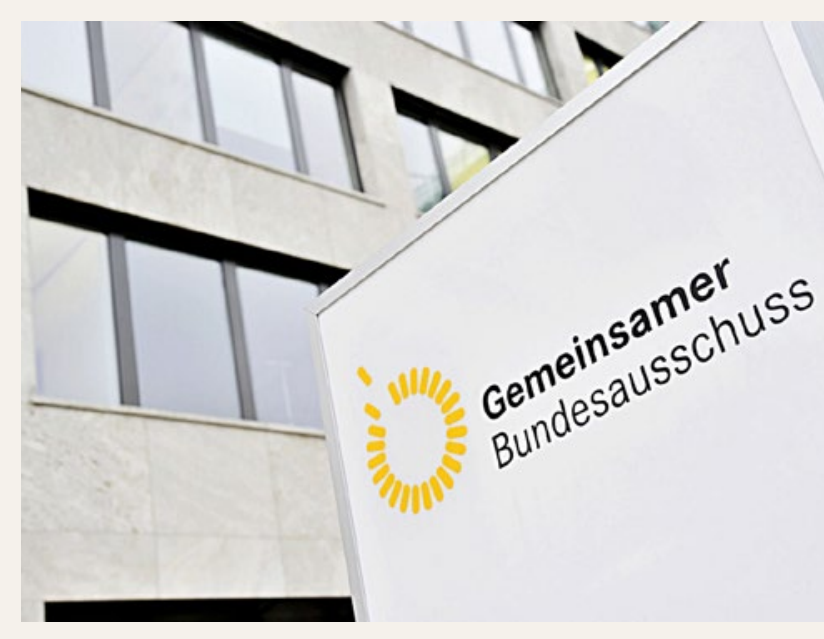

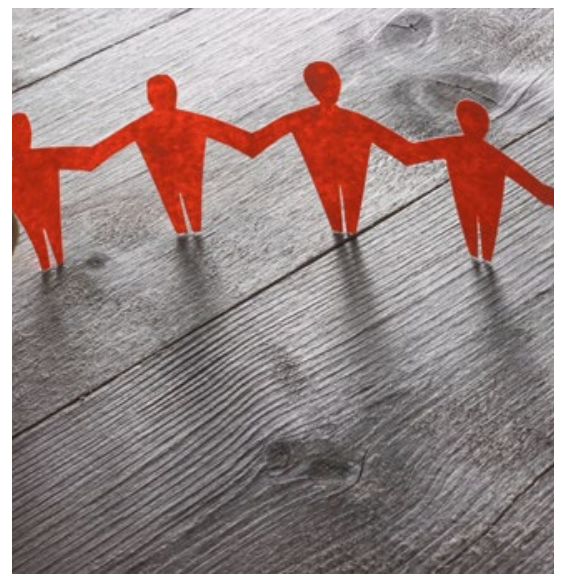

\section{ARZTNETZE VERBESSERN VERSORGUNG}

Regionale Zusammenarbeit-- Neue Kooperationsformen von Praxen stehen im Mittelpunkt vieler Überlegungen im Gesundheitsbereich. Über die Chancen und Aufgaben von Arztnetzen sprachen in diesem Zusammenhang Bundestagsabgeordnete und Vertreter der Agentur Deutscher Arztnetze (ADA) in Berlin. Regionale Arztnetze sind Zusammenschlüsse von Ärzten, um zum einen durch die Zusammenarbeit eine qualitativ bessere Versorgung von Patienten zu erreichen, zum anderen aber auch die eigene berufliche und wirtschaftliche Situation von Einzelpraxen zu optimieren und den demografischen Entwicklungen anzupassen. Nach Angaben der ADA haben Arztnetze seit der Möglichkeit zur Anerkennung durch die Kassenärztlichen Vereinigungen einen raschen Aufschwung erlebt. Rund 7.500 Ärzte arbeiten demnach deutschlandweit in solchen regionalen Netzen. Der Vorstandsvorsitzende der Agentur Deutscher Arztnetze, Veit Wambach, betonte die Rolle der niedergelassenen Ärzte. Auch in der Abgrenzung zu Medizinischen Versorgungszentren (MVZ) machte er deutlich: „Die ärztliche Leitung der Praxisnetze ist selbstverständlich, Kapitalinteressen dürfen niemals bestimmend sein. “ Für Drittinteressen habe Freiberuflichkeit keinen Platz. In der Diskussion sprachen Vertreter von CDU/CSU, FDP, Bündnis 90/Die Grünen und Linke über die zukünftigen Herausforderungen von guter Patientenversorgung und betonten einhellig, dass ärztliche Kooperation gerade in ländlichen Gebieten dringend notwendig sei. Die Politik könne dazu allerdings nur die Rahmenbedingungen und Leitplanken einziehen. Die Selbstgestaltungskraft der Selbstverwaltung sei gefragt, sagte Georg Kippels (CDU). SAS

\section{FINANZRESERVEN DER KASSEN STEIGEN WEITER}

Hoher Überschuss-- Die gesetzlichen Krankenkassen haben in den ersten drei Quartalen des Jahres 2017 einen Überschuss von rund 2,52 Milliarden Euro erzielt. Die Überschussentwicklung hat sich damit weiter beschleunigt. Die Finanzreserven der Kassen stiegen bis Ende September auf rund 18,6 Milliarden Euro, wie das Bundesgesundheitsministerium (BMG) mitteilte. Die durchschnittliche Finanzreserve sämtlicher Krankenkassen beträgt knapp eine Monatsausgabe und liegt damit fast viermal so hoch wie die gesetzlich vorgesehene Mindestreserve. Auf Basis der bisherigen Finanzentwicklung und der Erwartungen des Schätzerkreises für 2017 und für 2018 spreche alles dafür, dass die gesetzlichen Krankenkassen ihre Überschüsse auch im laufenden Jahr ausbauen können und die „nötigen Finanzspielräume für gute Leistungen bei attraktiven Beiträgen für die Versicherten“ haben. „Es liegt nun in der Hand der einzelnen Krankenkassen, diese Spielräume im Sinne der Versicherten auszuschöpfen“, sagte Bundesgesundheitsminister Hermann Gröhe. 